DESY 99-076

ISSN $0418-9833$

June 1999

\title{
Towards the Theory of Diffractive DIS*
}

\author{
W. Buchmüller \\ Deutsches Elektronen-Synchrotron DESY, 22603 Hamburg, Germany
}

\begin{abstract}
The large rapidity gap events, observed at HERA, have changed considerably our physical picture of deep inelastic scattering during the past years. We review the present theoretical understanding of diffractive DIS with emphasis on the close relation to inclusive DIS. This includes success and limitations of the leading twist description, the connection between diffractive and inclusive parton distributions in the semiclassical approach, the colour structure of the proton and comparison with data. The progress report concludes with a list of open questions.
\end{abstract}

*Presented at New Trends in HERA Physics 1999, Ringberg Workshop, June 1999 


\section{Inclusive and diffractive DIS}

The intriguing phenomenon of the frequent appearance of large rapidity gaps in electron proton collisions at HERA [1] has changed our physical picture of deep inelastic scattering (DIS) to a large extent. The large rapidity gap events are very difficult to understand in the parton model where the struck quark is expected to break up the proton leading to a continuous flow of hadrons between the current jet and the proton remnant.

To develop a physical picture of diffractive DIS [2] it is convenient to view the scattering process in the proton rest frame. In this frame the virtual photon fluctuates into partonic states $q \bar{q}, q \bar{q} g, \ldots$ which then scatter off the proton. From the leading twist contributions to inclusive and diffractive structure functions one obtains the parton distribution functions in a frame where the proton moves fast. This connection holds for diffractive as well as non-diffractive processes.

In the following we shall review the present status of our theoretical understanding of diffractive DIS with emphasis on the close analogy to inclusive DIS. This is appropriate since diffractive DIS is dominated by the leading twist contribution, which has been one of the most surprising aspects of the large rapidity gap events. The scattering of the partonic fluctuations of the photon off the proton will be treated in the semiclassical approach. After a comparison of theoretical predictions with data we shall conclude with a discussion of some open questions.

\section{Inclusive DIS}

Inclusive deep inelastic scattering [3] is characterized by the kinematic variables

$$
Q^{2}=-q^{2}, \quad W^{2}=(q+P)^{2}, \quad x=\frac{Q^{2}}{Q^{2}+W^{2}}
$$

where $q$ and $P$ are the momenta of the virtual photon and the proton, respectively. The cross section is determined by the hadronic tensor,

$$
\begin{aligned}
W_{\mu \nu}(P, q) & =\frac{1}{4 \pi} \sum_{X}\left\langle P\left|J_{\nu}(0)\right| X\right\rangle\left\langle X\left|J_{\mu}(0)\right| P\right\rangle(2 \pi)^{4} \delta\left(P-P_{X}\right) \\
& =\left(-g_{\mu \nu}+\frac{q_{\mu} q_{\nu}}{q^{2}}\right) F_{1}\left(x, Q^{2}\right)+\frac{1}{\nu}\left(P_{\mu}-\frac{\nu}{q^{2}} q_{\mu}\right)\left(P_{\nu}-\frac{\nu}{q^{2}} q_{\nu}\right) F_{2}\left(x, Q^{2}\right)
\end{aligned}
$$

Here $J_{\mu}(x)$ is the electromagnetic current, $\nu=q \cdot P$, and spin averaging has been implicitly assumed.

The structure functions are a sum of leading twist and of higher twist contributions which are suppressed by powers of $Q^{2}$,

$$
F_{i}\left(x, Q^{2}\right)=F_{i}^{(L T)}\left(x, Q^{2}\right)+\frac{F_{i}^{(H T)}\left(x, Q^{2}\right)}{Q^{2}}+\ldots
$$


The leading twist term is dominant for $Q^{2}$ above some value $Q_{0}^{2}$, which is not very well known and frequently chosen to be $\mathcal{O}\left(1 \mathrm{GeV}^{2}\right)$. However, higher twist contributions are known to be important for hadronic energies $W^{2} \leq 4 \mathrm{GeV}^{2}$ [ [⿴囗十).

The structure functions $F_{i}^{(L T)}\left(x, Q^{2}\right)$ can be expressed in terms of process independent parton distribution functions,

$$
F_{i}^{(L T)}\left(x, Q^{2}\right) \rightarrow f_{i}\left(x, \mu^{2}\right)=q\left(x, \mu^{2}\right), g\left(x, \mu^{2}\right)
$$

which depend on $x$ and on the factorization scale $\mu^{2}$. At small $x$, the quark distribution is assumed to be the same for all light flavours. The parton distribution functions $f_{i}\left(x, \mu^{2}\right)$ obey the perturbative QCD evolution equations [5],

$$
\mu^{2} \frac{\partial}{\partial \mu^{2}} f_{i}\left(x, \mu^{2}\right)=\frac{\alpha_{s}}{2 \pi} \int_{x}^{1} \frac{d y}{y} P_{i j}\left(\frac{x}{y}\right) f_{i}\left(y, \mu^{2}\right),
$$

where $P_{i j}(z)$ are the Altarelli-Parisi splitting functions. The parton distributions can be directly expressed in terms of the quark and gluon field operators. For instance, the quark distribution is given by

$$
\begin{aligned}
q\left(x, \mu^{2}\right)= & \frac{1}{4 \pi} \int d x_{-} e^{-i x P_{+} x_{-} / 2} \sum_{X} \\
& \left\langle P\left|\bar{q}\left(0, x_{-}, 0_{\perp}\right) U\left(x_{-}, \infty\right)\right| X\right\rangle \gamma_{+}\left\langle X\left|U(\infty, 0) q\left(0,0,0_{\perp}\right)\right| P\right\rangle,
\end{aligned}
$$

where $U(a, b)$ is the colour matrix

$$
U(a, b)=P \exp \left(-\frac{i}{2} \int_{b}^{a} d y_{-} A_{+}\left(0, y_{-}, 0_{\perp}\right)\right) .
$$

This definition can be used as a starting point of a theoretical non-perturbative evaluation of the quark distribution.

\section{Diffractive DIS}

Diffractive DIS can be discussed in close analogy to inclusive DIS. There are two more kinematical variables which charaterize the diffractively scattered proton: the invariant momentum transfer $t$ and the fraction $\xi$ of lost longitudinal momentum. A complete set of variables is

$$
t=\left(P-P^{\prime}\right)^{2}, \quad \xi \equiv x_{\mathbb{P}}, \quad Q^{2}=-q^{2}, \quad M^{2}=(q+\xi P)^{2}, \quad \beta=\frac{Q^{2}}{Q^{2}+M^{2}} .
$$

Compared to inclusive DIS, the diffractive mass $M$ plays the role of the total hadronic mass $W$, and $\beta$ corresponds to $x$.

The hadronic tensor for diffractive DIS,

$$
W_{\mu \nu}^{D}\left(P, P^{\prime}, q\right)=\frac{1}{4 \pi} \sum_{X}\left\langle P\left|J_{\nu}(0)\right| X ; P^{\prime}\right\rangle\left\langle X ; P^{\prime}\left|J_{\mu}(0)\right| P\right\rangle(2 \pi)^{4} \delta\left(P-P^{\prime}-P_{X}\right)
$$




$$
\begin{aligned}
=( & \left.-g_{\mu \nu}+\frac{q_{\mu} q_{\nu}}{q^{2}}\right) F_{1}^{D(4)}\left(t, \xi, \beta, Q^{2}\right) \\
& +\frac{1}{\nu}\left(P_{\mu}-\frac{\nu}{q^{2}} q_{\mu}\right)\left(P_{\nu}-\frac{\nu}{q^{2}} q_{\nu}\right) F_{2}^{D(4)}\left(t, \xi, \beta, Q^{2}\right)+\ldots,
\end{aligned}
$$

defines the diffractive structure functions $F_{i}^{D(4)}\left(t, \xi, \beta, Q^{2}\right)$. Integration over $t$, which is dominated by small $|t|$ for diffractive scattering, yields the extensively studied structure function

$$
F_{2}^{D(3)}\left(\xi, \beta, Q^{2}\right)=\int d t F_{2}^{D(4)}\left(t, \xi, \beta, Q^{2}\right)
$$

Also the diffractive structure functions have contributions of leading and higher twist,

$$
F_{i}^{D(3)}\left(\xi, \beta, Q^{2}\right)=F_{i}^{D(3, L T)}\left(\xi, \beta, Q^{2}\right)+\frac{F_{i}^{D(3, H T)}\left(\xi, \beta, Q^{2}\right)}{Q^{2}}+\ldots
$$

Again it is unclear above which value of $Q_{0}^{2}$ the leading twist part dominates. At small $x, W^{2} \simeq Q^{2} / x$ should be large enough, whereas the lower bound on $M^{2}$ is an open question. Our phenomenological analysis in the next section will show that the leading twist description breaks down at $M_{0}^{2} \simeq 4 \mathrm{GeV}^{2}$. This again demonstrates that in diffractive DIS $M^{2}$ plays a role analogous to $W^{2}$ in inclusive DIS.

For diffractive DIS factorization holds like for inclusive DIS [6]. The diffractive structure functions $F_{i}^{D(3, L T)}\left(\xi, \beta, Q^{2}\right)$ can be expressed in terms of 'fracture functions' [7], or 'diffractive parton distributions' [8],

$$
F_{i}^{D(3, L T)}\left(\xi, \beta, Q^{2}\right) \rightarrow \frac{d f_{i}\left(\xi, \beta, \mu^{2}\right)}{d \xi}=\frac{d q\left(\xi, \beta, \mu^{2}\right)}{d \xi}, \frac{d g\left(\xi, \beta, \mu^{2}\right)}{d \xi}
$$

which depend on $\xi, \beta$ and the factorization scale $\mu^{2}$. The diffractive parton distribution functions $d f_{i}\left(\xi, \beta, \mu^{2}\right) / d \xi$ also obey the perturbative QCD evolution equations,

$$
\mu^{2} \frac{\partial}{\partial \mu^{2}} \frac{d f_{i}\left(\xi, \beta, \mu^{2}\right)}{d \xi}=\frac{\alpha_{s}}{2 \pi} \int_{\beta}^{1} \frac{d b}{b} P_{i j}\left(\frac{\beta}{b}\right) \frac{d f_{i}\left(\xi, b, \mu^{2}\right)}{d \xi} .
$$

Note that the evolution takes now place in $\beta$ and $Q^{2} ; \xi$ merely acts as a parameter. The physical reason for this is intuitively clear: for an arbitrary DIS event the invariant hadronic mass is $W$, and the quark which couples to the virtual photon can be radiated by a parton whose fraction of the proton momentum varies from 1 to $x=Q^{2} /\left(Q^{2}+W^{2}\right)$. In a diffractive event, the diffractive invariant mass is $M$. Hence, $W$ is replaced by $M$, and the quark which couples to the photon can be radiated by a parton whose fraction of the momentum $\xi P$ varies from 1 to $\beta=Q^{2} /\left(Q^{2}+M^{2}\right)$. Formally, Eq. (13) follows from the fact that ultraviolet divergencies and renormalization are the same for inclusive and diffractive parton distribution functions [9]. This is apparent from a comparison of the corresponding operator definitions. The diffractive quark distribution, for instance, is given by [9],

$$
\begin{aligned}
\frac{d q\left(\xi, \beta, \mu^{2}\right)}{d \xi}= & \frac{1}{64 \pi^{3}} \int d t \int d x_{-} e^{-i x P_{+} x_{-} / 2} \sum_{X} \\
& \left\langle P\left|\bar{q}\left(0, x_{-}, \overrightarrow{0}\right) U\left(x_{-}, \infty\right)\right| X ; P^{\prime}\right\rangle \gamma_{+}\left\langle X ; P^{\prime}|U(\infty, 0) q(0,0, \overrightarrow{0})| P\right\rangle .
\end{aligned}
$$




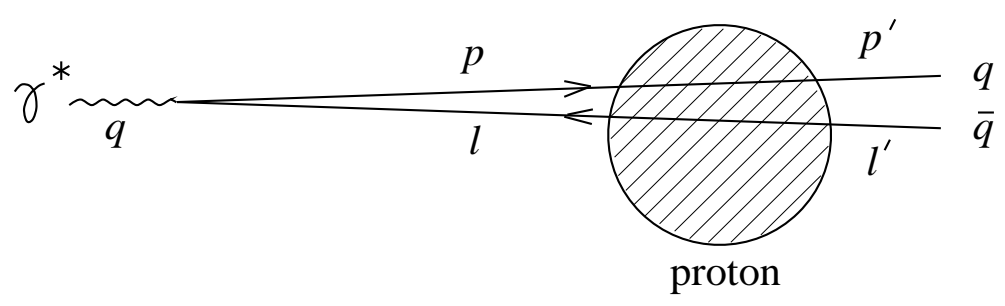

Figure 1: Diffractive or non-diffractive DIS in the proton rest frame; the proton is viewed as a superposition of colour fields with size $1 / \Lambda$.

Assuming 'Regge factorization' for the diffractive quark and gluon distribution functions yields the Ingelman-Schlein model of hard diffractive scattering [10 which can also be applied to deep inelastic scattering [11].

The physical interpretation of the diffractive parton distributions is analogous to the interpretation of the inclusive distributions. The function $d f\left(\xi, b, \mu^{2}\right) / d \xi$ is a conditional probability distribution. It describes the probability density to find a parton $f$, carrying a fraction $\xi b$ of the proton momentum, under the condition that the proton has lost a fraction $\xi$ of its momentum in the scattering process.

The formal definition of diffractive parton distributions tells us very little about their properties, although, comparing Eqs. (6) and (14), one may expect that diffractive DIS is a leading twist effect. However, the important physics question concerns the relation between the two types of distribution functions,

$$
f_{i}\left(x, \mu^{2}\right) \longleftrightarrow \frac{d f_{i}\left(\xi, \beta, \mu^{2}\right)}{d \xi} \quad ?
$$

Both kinds of parton distributions represent non-perturbative properties of the proton and are therefore not accessible to perturbation theory. Still, one may hope that at small $x$, i.e. large hadronic energies $W$, some simple relations between inclusive and diffractive deep inelastic scattering may exist. In the following section we shall describe a picture of hadrons at small $x$ where this is indeed the case.

\section{Semiclassical approach}

The phenomenon of the large rapidity gap events in DIS is very difficult to understand within the parton model. Naively, one would expect that the struck quark will always break up the proton, which should lead to a flow of hadrons between the current jet and the proton remnant without large gaps in rapidity.

The connection between diffractive DIS and ordinary, non-diffractive DIS can be most easily understood in the proton rest frame which has frequently been used in the early days of DIS, almost 30 years ago. In this frame, DIS appears as the scattering of partonic fluctuations of the photon, $q \bar{q}, q \bar{q} g$ etc., off the proton. In the semiclassical approach [12 the proton is viewed as a superposition of colour fields of size $1 / \Lambda$ in DIS at small $x$, i.e. at high $\gamma^{*} p$ center-of-mass energies. The simplest partonic fluctuation is 
a quark-antiquark pair (cf. Fig. 1). Penetrating the proton, quark and antiquark change their colour. If the $q \bar{q}$ pair leaves the proton in a colour singlet configuration, it can fragment independently of the proton remnant yielding a diffractive event. A $q \bar{q}$ pair in a colour octet state will build up a flux tube together with the proton remnant, whose breakup will lead to an ordinary non-diffractive event.

The scattering amplitude for both types of events is determined by a single nonperturbative quantity, $\operatorname{tr} W_{x_{\perp}}\left(y_{\perp}\right)$. Here $x_{\perp}$ and $x_{\perp}+y_{\perp}$ are the transverse positions where quark and antiquark penetrate the colour field of the proton. The function

$$
W_{x_{\perp}}\left(y_{\perp}\right)=U\left(x_{\perp}\right) U^{\dagger}\left(x_{\perp}+y_{\perp}\right)-1
$$

with

$$
U\left(x_{\perp}\right)=P \exp \left(-\frac{i}{2} \int_{-\infty}^{\infty} d x_{-} A_{+}\left(0, x_{-}, x_{\perp}\right)\right),
$$

is essentially a closed Wilson loop through the corresponding section of the proton, which measures an integral of the proton colour field strength.

Diffractive DIS requires a colour singlet pair in the final state. Hence the scattering amplitude is $\propto \operatorname{tr} W_{x_{\perp}}\left(y_{\perp}\right)$ and the diffractive cross section takes the form,

$$
d \sigma^{D} \propto \int_{x_{\perp}} \ldots\left|\ldots \operatorname{tr} W_{x_{\perp}}\left(y_{\perp}\right) \ldots\right|^{2}
$$

The inclusive cross section is obtained by summing over all colours, which yields

$$
\begin{aligned}
d \sigma^{i n c l} & \propto \int_{x_{\perp}} \ldots \operatorname{tr}\left(W_{x_{\perp}}\left(y_{\perp}\right) W_{x_{\perp}}^{\dagger}\left(y_{\perp}\right)\right) \ldots \\
& \propto \int_{x_{\perp}} \ldots \operatorname{tr} W_{x_{\perp}}\left(y_{\perp}\right) \ldots
\end{aligned}
$$

where the last equation follows from the unitarity of the matrix $U\left(x_{\perp}\right)$.

From Eqs. (18) and (19) one immediately derives the properties of Bjorken's aligned jet model [13]. For small quark-antiquark separations one has,

$$
\int_{x_{\perp}} \operatorname{tr} W_{x_{\perp}}\left(y_{\perp}\right) \propto y_{\perp}^{2}
$$

Hence, since all kinematical factors are the same for $d \sigma^{D}$ and $d \sigma^{i n c l}$, small $q \bar{q}$ pairs are suppressed in diffractive DIS. For large pairs of size $1 / \Lambda$, the transverse momentum $l_{\perp}^{\prime}$ and the longitudinal momentum fractions $\alpha$ and $1-\alpha$ are

$$
l_{\perp}^{\prime} \sim \Lambda, \quad \alpha \sim \frac{\Lambda^{2}}{Q^{2}}, \quad 1-\alpha \simeq 1
$$

These are the asymmetric, aligned jet configurations [13] which dominate diffractive DIS. 


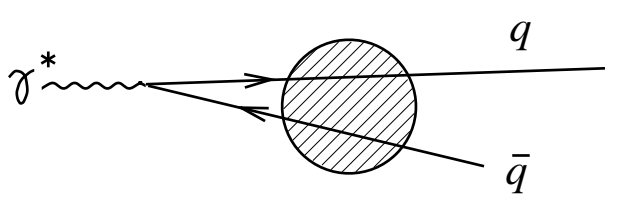

a)

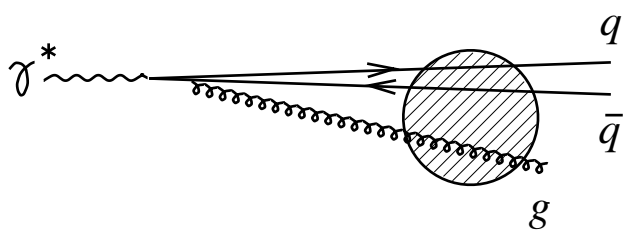

b)
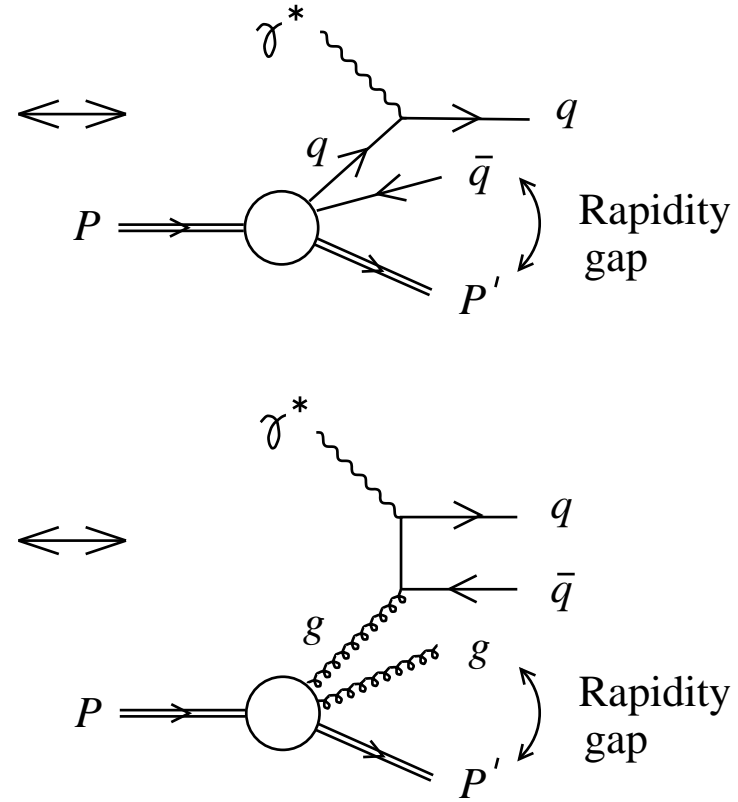

Figure 2: Diffractive DIS in the proton rest frame (left) and the Breit frame (right); asymmetric quark fluctuations correspond to diffractive quark scattering, asymmetric gluon fluctuations to diffractive boson-gluon fusion.

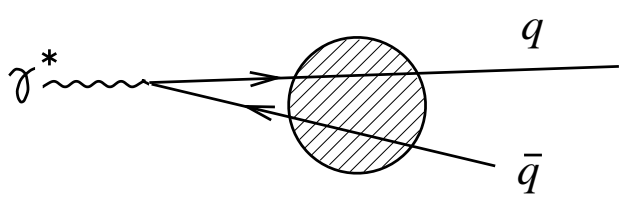

a)

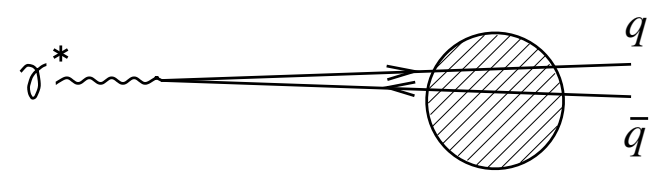

b)
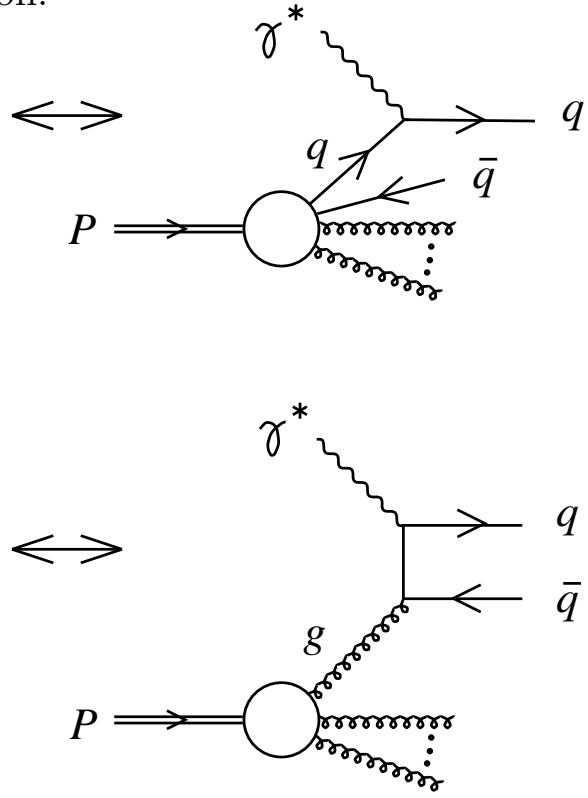

Figure 3: Inclusive DIS in the proton rest frame (left) and the Breit frame (right); asymmetric fluctuations correspond to quark scattering (a), symmetric fluctuations to boson-gluon fusion (b). 


\section{Diffractive and inclusive parton distributions}

In the semiclassical approach the evaluation of inclusive and diffractive structure functions is straightforward, in principle. One has to calculate the scattering amplitudes for the production of $q \bar{q}, q \bar{q} g$... configurations [14 in an external colour field, analogous to the production of $\mu^{+} \mu^{-}$pairs in an external electromagnetic field [15], treat the interaction of the fast partons with the non-abelian colour field in the eikonal approximation [16], and finally integrate over all target colour fields.

The result for the leading twist part can be expressed in terms of diffractive parton distributions [17]. For the transverse structure function, for instance, one finds to leading order in the QCD coupling,

$$
\begin{gathered}
F_{T}^{D}\left(\xi, \beta, Q^{2}\right)=2 e_{q}^{2} x \int_{\beta}^{1} \frac{d b}{b}\left\{\left(\delta(1-z)+\frac{\alpha_{s}}{2 \pi}\left(P_{q q}(z) \ln \frac{Q^{2}}{\mu^{2}}+\ldots\right)\right) \frac{d q\left(b, \xi, \mu^{2}\right)}{d \xi}\right. \\
\left.+\frac{\alpha_{s}}{2 \pi}\left(P_{q g}(z) \ln \frac{Q^{2}}{\mu^{2}}+\ldots\right) \frac{d g\left(b, \xi, \mu^{2}\right)}{d \xi}\right\}
\end{gathered}
$$

where $z=\beta / b$, and $C_{F}$ and $T_{F}$ are the usual colour factors. This expression is completely analogous to the well known result for the inclusive structure function $F_{T}\left(x, Q^{2}\right)$. In the diffractive case, $\beta$ plays the role of $x$, whereas $\xi$ only acts as a parameter. From Eq. (22) it is obvious that, as anticipated, the diffractive parton distributions satisfy the perturbative QCD evolution equations (13).

The diffractive quark and gluon distributions have been determined in [17]. In terms of Wilson loops in coordinate space, the quark distribution can be expressed as follows,

$$
\begin{aligned}
\frac{d q\left(\xi, b, \mu^{2}\right)}{d \xi}= & \frac{2 b}{\xi^{2}(1-b)^{3}} \int \frac{d^{2} l_{\perp}^{\prime} l_{\perp}^{\prime}}{(2 \pi)^{6} N_{c}} \int_{y_{\perp}, y_{\perp}^{\prime}} e^{i l_{\perp}^{\prime}\left(y_{\perp}-y_{\perp}^{\prime}\right)} \frac{y_{\perp} y_{\perp}^{\prime}}{y y^{\prime}} \\
& \times K_{1}(y N) K_{1}\left(y^{\prime} N\right) \int_{x_{\perp}} \operatorname{tr} W_{x_{\perp}}\left(y_{\perp}\right) \operatorname{tr} W_{x_{\perp}}^{\dagger}\left(y_{\perp}^{\prime}\right)
\end{aligned}
$$

where $N_{c}$ is the number of colours and $N^{2}=l_{\perp}^{\prime 2} \frac{b}{1-b}$.

It is very instructive to compare diffractive DIS in the proton rest frame and in the Breit frame (cf. Fig. 2). The number of partons in the final state is the same, of course, in both frames. Note, however, that the virtual parton connected to the proton changes it's direction. It appears incoming in the proton rest frame and outgoing in the Breit frame. Diffractive quark and gluon distributions correspond to asymmetric $q \bar{q}$ and $q \bar{q} g$ fluctuations with a slow antiquark and gluon, respectively.

Inclusive parton distributions can be calculated in a similar way. The inclusive quark distribution is again given by the asymmetric $q \bar{q}$ configuration (cf. Fig. 3), just with arbitrary colours in the final state. A special role is played by the inclusive gluon distribution. It is related to small symmetric $q \bar{q}$ pairs which probe the colour field of the proton directly (cf. Fig. 3). Contrary to all other parton distributions, the inclusive gluon distribution [18, 


$$
\begin{aligned}
x g\left(x, Q^{2}\right) & =\frac{3 \pi}{\alpha_{s} e_{q}^{2}} \cdot \frac{\partial F_{T}\left(x, Q^{2}\right)}{\partial \ln Q^{2}} \\
& =\frac{1}{2 \pi^{2} \alpha_{s}} \int_{x_{\perp}} \operatorname{tr}\left(\partial_{y_{\perp}} W_{x_{\perp}}(0) \partial_{y_{\perp}} W_{x_{\perp}}^{\dagger}(0)\right)=\mathcal{O}\left(\frac{1}{\alpha_{s}}\right),
\end{aligned}
$$

is enhanced by an inverse power of $\alpha_{s}$ in the semiclassical approach. This is the reason why diffractive DIS is suppressed. Note that the gluon distribution is directly related to the cross section for a small $q \bar{q}$ pair with transverse size $y$ [19],

$$
\sigma_{q \bar{q}}\left(y ; x, Q^{2}\right)=\frac{\pi^{2}}{3} \alpha_{s} x g\left(x, Q^{2}\right) y^{2}+\mathcal{O}\left(y^{4}\right) \text {. }
$$

\section{Integration over the target gluon fields}

So far we have expressed diffractive and inclusive parton distributions in terms of Wilson loops which integrate the gluon field strength in the area between the trajectories of two fast colour charges penetrating the proton. The integration over the gluon field configurations of the target is a complicated operation depending on the full details of the non-perturbative hadronic state. However, in the special case of a very large target, a quantitative treatment becomes possible under minimal additional assumptions. The reason is that the large size of a hadronic target, realized, e.g., in an extremely heavy nucleus, introduces a new hard scale [20]. From the target rest frame point of view, this means that the typical transverse size of the partonic fluctuations of the virtual photon remains perturbative [21], thus justifying the omission of higher Fock states in the semiclassical calculation.

Within this framework, it is natural to introduce the additional assumption that the gluonic fields encountered by the partonic probe in distant regions of the target are not correlated. Thus, one arrives at the situation depicted in Fig. 田, where a colour dipole passes a large number of regions, each one of size $\sim 1 / \Lambda$, with mutually uncorrelated colour fields $A_{1} \ldots A_{n}$.

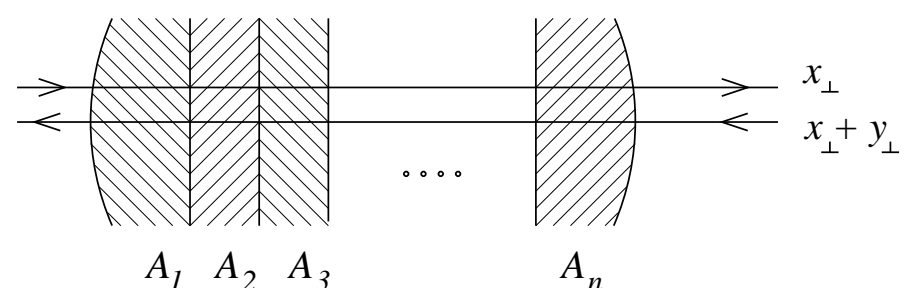

Figure 4: Colour dipole travelling through a large hadronic target.

The crucial assumption that the fields in regions $1 \ldots n$ are uncorrelated is implemented by writing the integral over all field configurations as

$$
\int_{A}=\int_{A_{1}} \cdots \int_{A_{n}}
$$


i.e., as a product of independent integrals. Here the appropriate weighting provided by the target wave functional is implicit in the symbol $\int_{A}$. For inclusive and diffractive parton distributions we need the two colour contractions for products of Wilson loops,

$$
\operatorname{tr}\left(W_{x_{\perp}}\left(y_{\perp}\right) W_{x_{\perp}}^{\dagger}\left(y_{\perp}\right)\right) \longleftrightarrow \frac{1}{N_{c}} \operatorname{tr} W_{x_{\perp}}\left(y_{\perp}\right) \operatorname{tr} W_{x_{\perp}}^{\dagger}\left(y_{\perp}\right)
$$

This relation, which provides the connection between inclusive and diffractive DIS, is the essence of the semiclassical approach.

Performing the integration over the colour fields one obtains in the large $N_{c}$ limit [18],

$$
\begin{aligned}
\int_{x_{\perp}} \int_{A} \operatorname{tr}\left(W_{x_{\perp}}\left(y_{\perp}\right) W_{x_{\perp}}^{\dagger}\left(y_{\perp}^{\prime}\right)\right) & =\Omega N_{c}\left(1-e^{-a y^{2}}-e^{-a y^{\prime 2}}+e^{-a\left(y_{\perp}-y_{\perp}^{\prime}\right)^{2}}\right), \\
\frac{1}{N_{c}} \int_{x_{\perp}} \int_{A} \operatorname{tr} W_{x_{\perp}}\left(y_{\perp}\right) \operatorname{tr} W_{x_{\perp}}^{\dagger}\left(y_{\perp}^{\prime}\right) & =\Omega N_{c}\left(1-e^{-a y^{2}}\right)\left(1-e^{-a y^{\prime 2}}\right),
\end{aligned}
$$

where $\Omega=\int d^{2} x_{\perp}$ is the geometric size of the target and $a$ plays the role of a saturation scale. Note that according to Eqs. (29) and (30) the diffractive structure function is not suppressed by a colour factor relative to the inclusive structure function, in contrast to the suggestion made in 22].

As an example, consider the inclusive quark distribution. From Eqs. (23), (28) and (29) one obtains, after changing the integration variable $\xi$ to $N^{2}$,

$$
x q\left(x, \mu^{2}\right)=\int_{x}^{1} d \xi \frac{d q\left(\xi, b=x / \xi, \mu^{2}\right)}{d \xi}=\int_{x_{\perp}} \int_{l_{\perp}^{\prime}} \frac{d x q\left(x, \mu^{2}\right)}{d^{2} x_{\perp} d^{2} l_{\perp}^{\prime}},
$$

with the unintegrated quark density

$$
\begin{aligned}
\frac{d x q\left(x, \mu^{2}\right)}{d^{2} x_{\perp} d^{2} l_{\perp}^{\prime}}= & \frac{N_{c}}{32 \pi^{6}} \int_{0}^{\mu^{2}} d N^{2} N^{2} \int_{y_{\perp}, y_{\perp}^{\prime}} e^{i l_{\perp}^{\prime}\left(y_{\perp}-y_{\perp}^{\prime}\right)} \frac{y_{\perp} y_{\perp}^{\prime}}{y y^{\prime}} \\
& \times K_{1}(y N) K_{1}\left(y^{\prime} N\right)\left(1-e^{-a y^{2}}-e^{-a y^{\prime 2}}+e^{-a\left(y_{\perp}-y_{\perp}^{\prime}\right)^{2}}\right) .
\end{aligned}
$$

This result has recently been obtained for the quark density in a large nucleus [23 by exponentiating the amplitude for a small $q \bar{q}$ pair scattering off a single nucleon, which is described by two-gluon exchange. The effect of the varying thickness of the nucleus has also been taken into account in [23], which makes the saturation scale $a$ dependent on the impact parameter $x_{\perp}$.

A Glauber type model with two-gluon exchange, similar in spirit to [24], has recently been used to study the effect of parton saturation in inclusive and diffractive DIS [25]. Although perturbative two-gluon exchange is a higher twist effect, the contribution from the soft region can be used as a model for inclusive and diffractive DIS [26, 27].

Particularly close to the semiclassical approach is the light cone hamiltonian approach to diffractive processes [28], which is also based on diffractive parton densities expressed in terms of expectation values of products of Wilson lines. For a hadronic target, modelled as a colour singlet which only couples to one flavour of heavy quarks, diffractive DIS is 

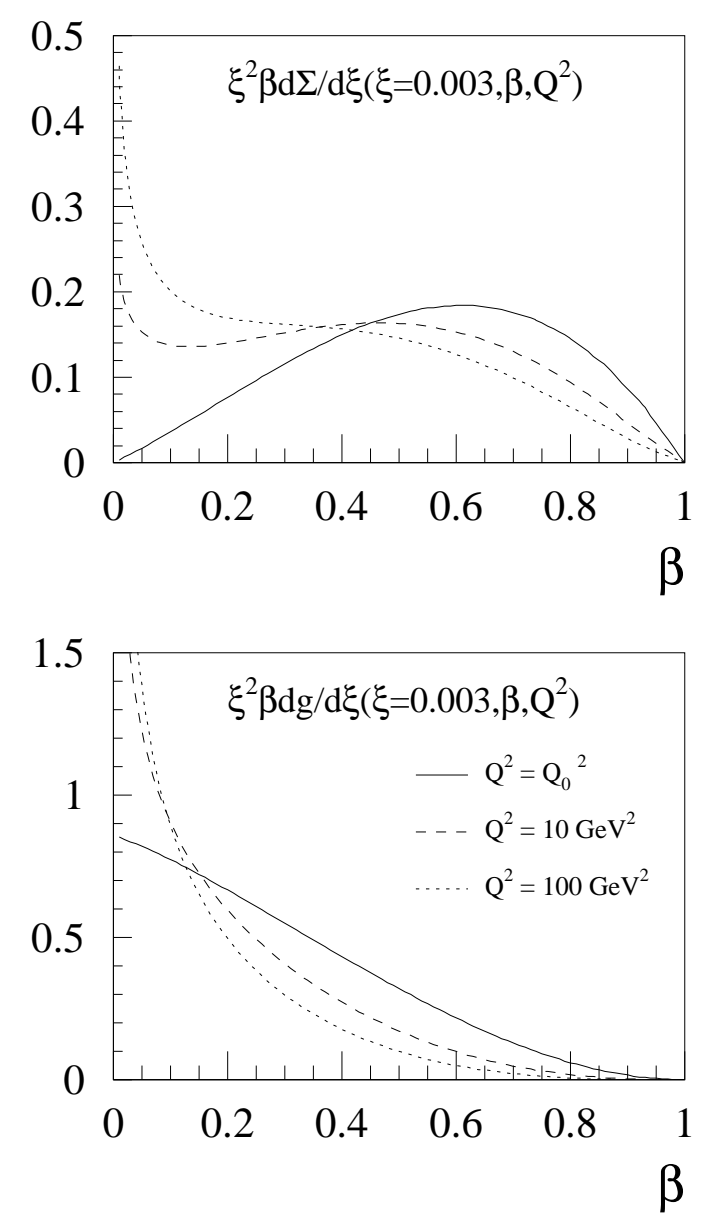

Figure 5: Diffractive quark and gluon distributions at the initial scale $Q_{0}^{2}$ and after $Q^{2}$ evolution. From 18].

dominated by two-gluon exchange. In the semiclassical approach, the proton is described by a superposition of colour fields. The role of classical colour fields in the case of high gluon densities has first been discussed by McLerran and Venugopalan in the case of a large nucleus [20].

\section{Comparison with data}

We now use the large hadronic target as a toy model for the proton. In case the proton can be viewed as an ensemble of regions with independently fluctuating colour fields, the model might even be realistic. We have explicitly verified that in the semiclassical approach inclusive and diffractive parton distributions satisfy the DGLAP evolution equations [5]. Hence, we can use the calculated quark and gluon distributions as nonperturbative input at some scale $Q_{0}^{2}$ and determine the distributions at larger $Q^{2}$ by means of the evolution equations. 

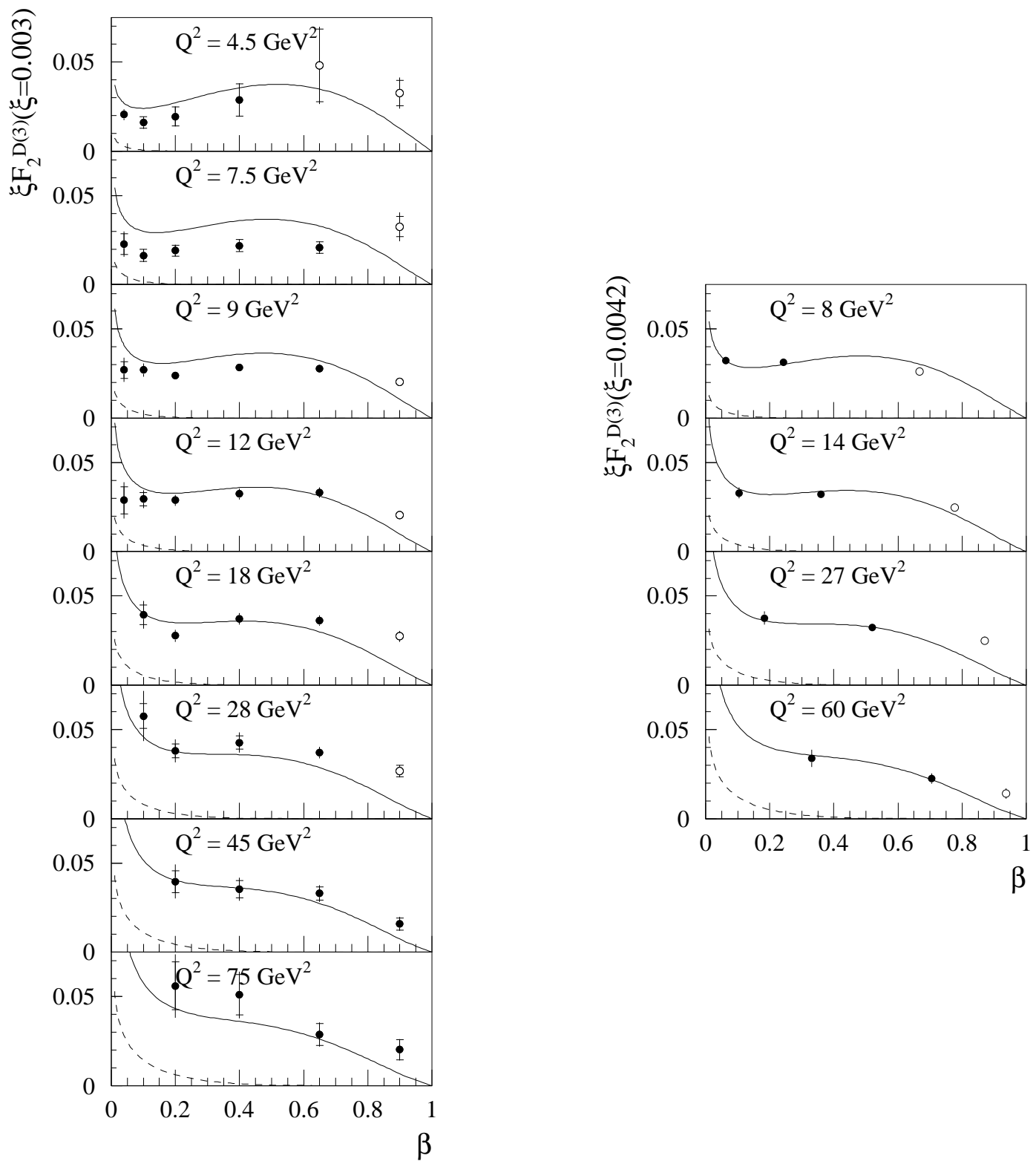

Figure 6: Dependence of the diffractive structure function $F_{2}^{D(3)}$ on $\beta$ and $Q^{2}$, compared to data from H1 (left) [29] and ZEUS (right) [30]. Open data points correspond to $M^{2} \leq 4 \mathrm{GeV}^{2}$. The charm content of the structure function is indicated as a dashed line. From [18]. 


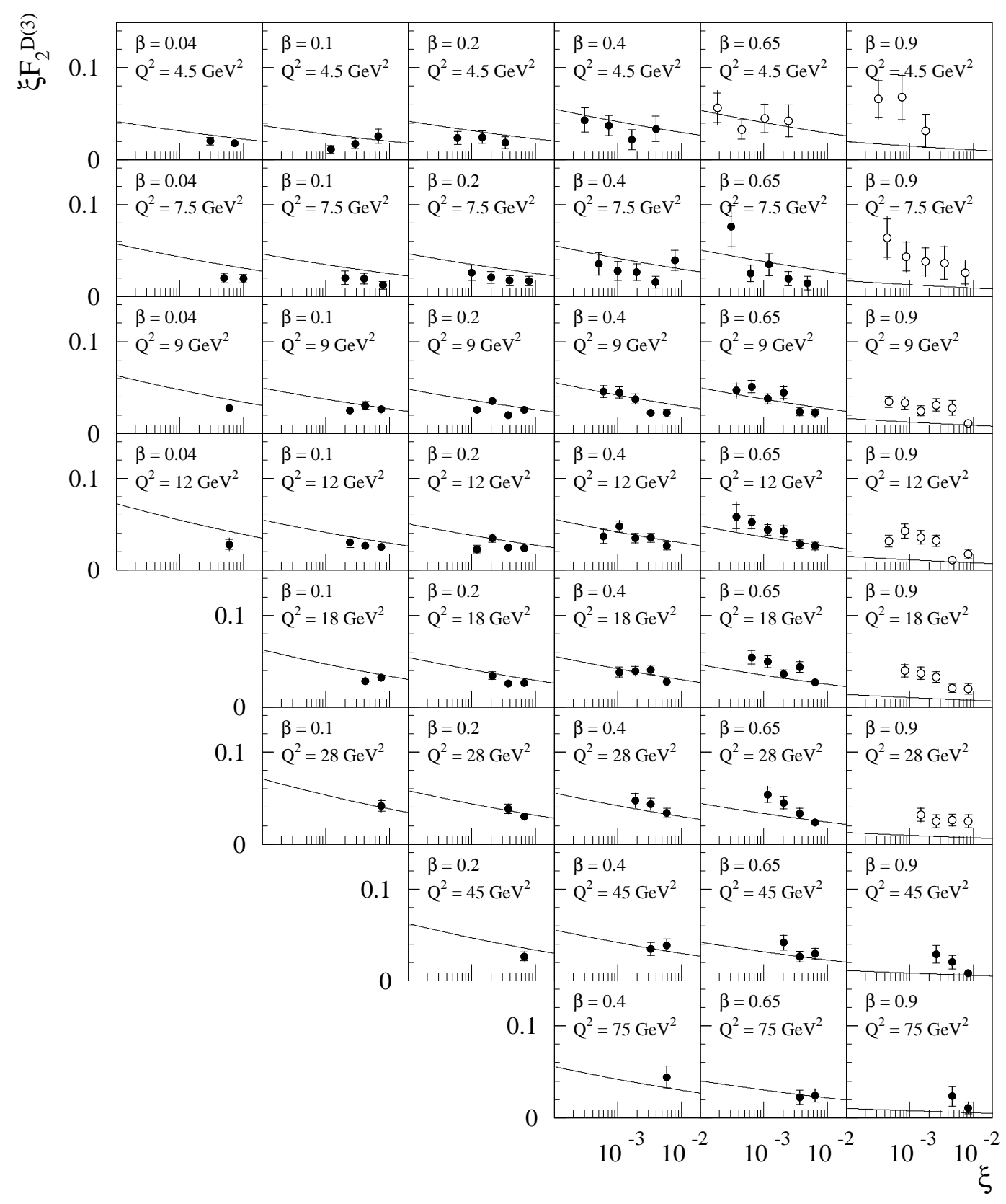

Figure 7: The diffractive structure function $F_{2}^{D(3)}\left(\xi, \beta, Q^{2}\right)$ at small $\xi$ computed in the semiclassical approach, using the fitted parameters given in the text. H1 data taken from [29]. The open data points correspond to $M^{2} \leq 4 \mathrm{GeV}^{2}$ and are not included in the fit. From [18]. 
For a given colour field, the semiclassical description of parton distribution functions always predicts an energy dependence corresponding to a classical bremsstrahlung spectrum: $q(x), g(x) \sim 1 / x$. One expects that, in a more complete treatment, a non-trivial energy dependence is induced since the integration over the soft target colour fields encompasses more and more modes with increasing energy of the probe [18]. At present we are unable to calculate this non-perturbative energy dependence from first principles. Instead, we choose to parametrize it in the form of a soft, logarithmic growth of the normalization of diffractive and inclusive parton distributions with the collision energy $\sim 1 / x$, consistent with the unitarity bound. This introduces one further parameter, $L$, into the model,

$$
\Omega \rightarrow \Omega(L-\ln x)^{2} .
$$

Including this energy dependence, one obtains the following compact expressions for inclusive and diffractive parton distributions [18],

$$
\begin{aligned}
x q\left(x, Q_{0}^{2}\right) & =\frac{a \Omega N_{c}(L-\ln x)^{2}}{3 \pi^{3}}\left(\ln \frac{Q_{0}^{2}}{a}-0.6424\right), \\
x g\left(x, Q_{0}^{2}\right) & =\frac{2 a \Omega N_{c}(L-\ln x)^{2}}{\pi^{2} \alpha_{s}\left(Q_{0}^{2}\right)}, \\
\frac{d q\left(\beta, \xi, Q_{0}^{2}\right)}{d \xi} & =\frac{a \Omega N_{c}(1-\beta)(L-\ln \xi)^{2}}{2 \pi^{3} \xi^{2}} f_{q}(\beta), \\
\frac{d g\left(\beta, \xi, Q_{0}^{2}\right)}{d \xi} & =\frac{a \Omega N_{c}^{2}(1-\beta)^{2}(L-\ln \xi)^{2}}{2 \pi^{3} \beta \xi^{2}} f_{g}(\beta) .
\end{aligned}
$$

These expressions are only applicable in the small- $x$ region, which we define by $x \leq \xi \leq$ 0.01 . The functions $f_{q, g}(\beta)$ are parameter free predictions. The model does not specify whether, in the diffractive case, the energy-dependent logarithm should be a function of $x$ or of $\xi$. However, both prescriptions differ only by terms proportional to $\ln \beta$, which can be disregarded in comparison with $\ln x$ or $\ln \xi$ in the small- $x$ region.

The above equations summarize our input distributions, depending on $a, \Omega, L$, and the input scale $Q_{0}^{2}$. At this order, the measured structure function $F_{2}$ coincides with the transverse structure function $F_{T}$. We assume all three light quark flavours to yield the same contribution, such that the singlet quark distribution is simply six times the quark distribution defined above, both in the inclusive and in the diffractive case,

$$
\Sigma\left(x, Q^{2}\right)=6 q\left(x, Q^{2}\right), \quad \frac{d \Sigma\left(\xi, \beta, Q^{2}\right)}{d \xi}=6 \frac{d q\left(\xi, \beta, Q^{2}\right)}{d \xi} .
$$

Valence quark contributions are absent in the semiclassical approach, which does not account for the exchange of flavour quantum numbers between the proton and the fast moving virtual photon state. Charm quarks are treated as massive quarks in the fixed flavour number scheme [32] (we use $\Lambda_{\mathrm{LO}, n_{f}=3}=144 \mathrm{MeV}, \alpha_{s}\left(M_{Z}\right)=0.118$, $\left.m_{c}=1.5 \mathrm{GeV}, m_{b}=4.5 \mathrm{GeV}, \mu_{c}=2 m_{c}\right)$. A fit to the data yields for the model parameters $Q_{0}^{2}=1.23 \mathrm{GeV}^{2}, L=8.16, \Omega=(712 \mathrm{MeV})^{-2}, a=(74.5 \mathrm{MeV})^{2}$. The starting scale $Q_{0}^{2}$ is in the region where one would expect the transition between perturbative 


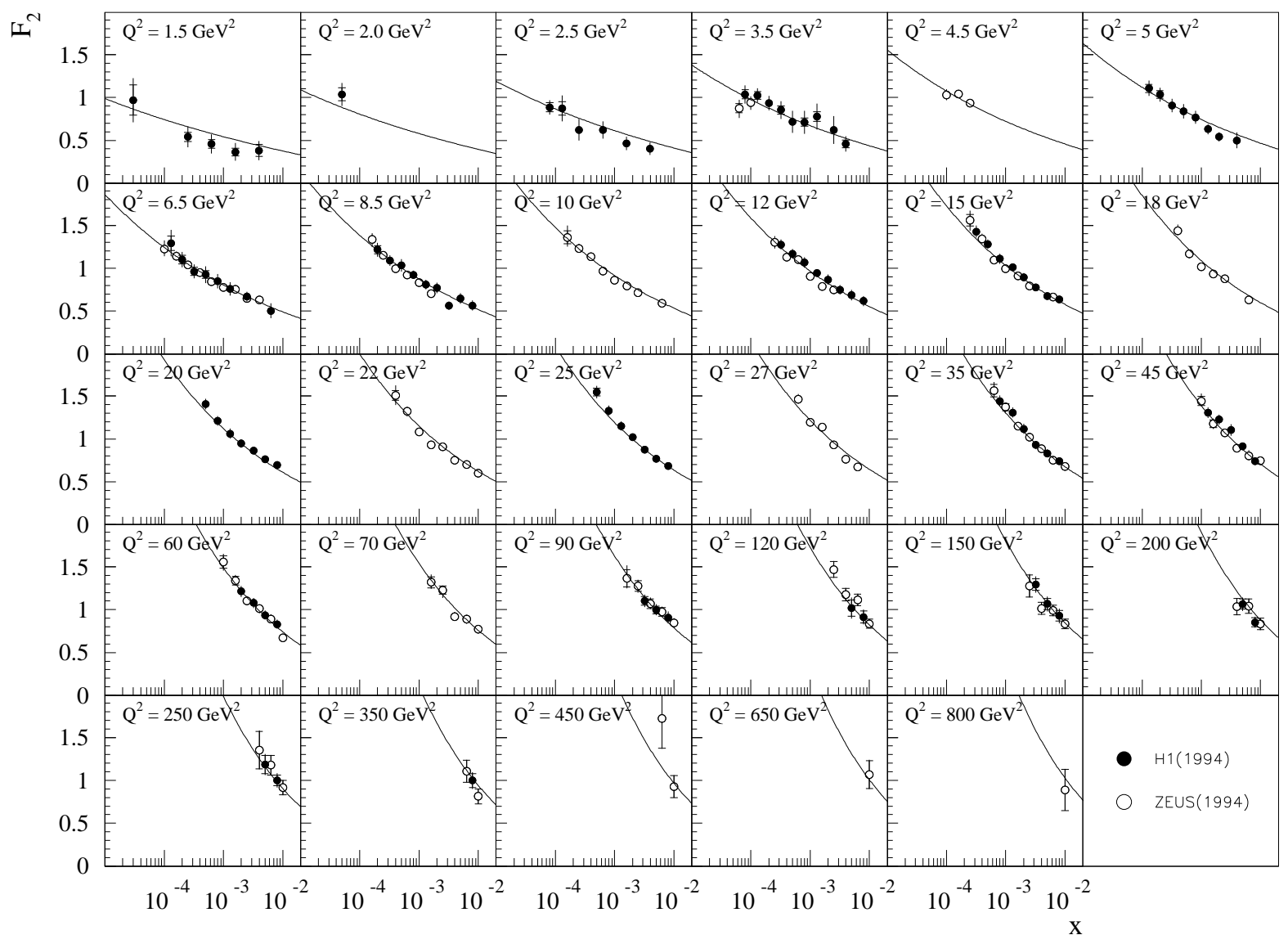

Figure 8: The inclusive structure function $F_{2}\left(x, Q^{2}\right)$ at small $x$ computed in the semiclassical approach, using the fitted parameters given in the text. Data taken from [31]. The data with $Q^{2}=1.5 \mathrm{GeV}^{2}$ are not included in the fit. From [18].

and non-perturbative dynamics to take place; the two other dimensionful parameters $\Omega L^{2}$ and $a$ are both of the order of typical hadronic scales.

The perturbative evolution of inclusive and diffractive structure functions is driven by the gluon distribution, which is considerably larger than the singlet quark distribution in both cases. The ratio of the inclusive singlet quark and gluon distributions can be read off from Eqs. (34) and (35). For the obtained fit parameters, it turns out that the inclusive gluon distribution is about twice as large as the singlet quark distribution.

The relative magnitude and the $\beta$ dependence of the diffractive distributions are completely independent of the model parameters. Moreover, their absolute normalization is, up to the slowly varying factor $1 / \alpha_{s}\left(Q_{0}^{2}\right)$, closely tied to the normalization of the inclusive gluon distribution.

Figure 5 displays the diffractive distributions for fixed $\xi=0.003$ and different values of $Q^{2}$. The $\beta$ dependences of the quark and the gluon distribution at $Q_{0}^{2}$ are substantially different: the asymmetric quark distribution $\beta d \Sigma / d \xi$ is peaked around $\beta \approx 0.65$, thus 
being harder than the symmetric distribution $\beta(1-\beta)$ suggested in [11]. The gluon distribution $\beta d g / d \xi$, on the other hand, approaches a constant for $\beta \rightarrow 0$ and falls off like $(1-\beta)^{2}$ at large $\beta$. In spite of the $(1-\beta)^{2}$ behaviour, gluons remain important even at large $\beta$, simply due to the large total normalization of this distribution (the $\beta$ integral over $\beta d g / d \xi$ at $Q_{0}^{2}$ is approximately three times the $\beta$ integral over $\left.\beta d \Sigma / d \xi\right)$. As a result, the quark distribution does not change with increasing $Q^{2}$ for $\beta \approx 0.5$ and is only slowly decreasing for larger values of $\beta$.

The dependence of the diffractive structure function on $\beta$ and $Q^{2}$ is illustrated in Fig. 6, where the predictions are compared with data from the H1 and ZEUS experiments [29, 30] at fixed $\xi$. Disregarding the large- $\beta$ region, the model gives a good description of the $\beta$ dependence of the diffractive structure function for all values of $Q^{2}$. It is remarkable that the qualitative features of the $\beta$ and $Q^{2}$ dependence are also correctly described by the perturbative approach of [28]. This indicates that the $\beta$ dependence of the diffractive structure function is to a large extent determined by the kinematics of the $q \bar{q}$ and $q \bar{q} g$ fluctuations and only partly sensitive to the details of the soft interaction with the proton. Also the $\xi$ dependence of the diffractive structure function is rather well described for $M^{2}>4 \mathrm{GeV}^{2}$, as demonstrated in Fig. 17. It has been demonstrated in [27] that higher twist contributions can account for the data at low $M^{2}$ (below 4 $\mathrm{GeV}^{2}$ ). This is analogous to the breakdown of the leading twist description of the inclusive structure functions, where it occurs for similar invariant hadronic masses, namely $W^{2} \lesssim 4 \mathrm{GeV}^{2}$ 四.

Finally, also the data of the $\mathrm{H} 1$ and ZEUS experiments on the inclusive structure function $F_{2}\left(x, Q^{2}\right)$ [31] are well reproduced by the model, as demonstrated by Fig. 8 .

\section{Open questions}

The theoretical work, stimulated by the observation of the large rapidity gap events at HERA, has led to a clear understanding of diffractive DIS as a leading twist phenomenon. Diffractive parton distribution functions can be defined completely analogous to inclusive parton distribution functions. Both kinds of distribution functions obey the perturbative QCD evolution equations. The parton distribution functions cannot be calculated perturbatively, any relation between them reflects a non-perturbative property of the proton. The leading twist description breaks down at $W^{2}<4 \mathrm{GeV}^{2}$ and $M^{2}<4$ $\mathrm{GeV}^{2}$ for inclusive and diffractive DIS, respectively.

A physical picture for diffractive DIS, and the relation to inclusive DIS, is most easily obtained in the proton rest frame where all DIS processes correspond to the scattering of partonic fluctuations of the virtual photon off the proton. In the semiclassical approach the proton is described by a superposition of colour fields. The qualitative properties of the $\beta$ dependence of the diffractive structure function are well reproduced by the scattering of colour dipoles off colour fields, which are generated either by a small colour dipole or by a large nucleus. This supports our general ideas about diffractive DIS. It will be interesting to see whether a more precise measurement of the $\beta$ spectrum will be quantitatively consistent with the idea of a colour field fluctuating independently in 
different sections of the proton.

In the semiclassical approach the proton colour field is assumed to be dominated by soft modes. Hence, diffractive and non-diffractive DIS events are kinematically very similar at small $x$, i.e. large hadronic energies $W$. This leads to an approximate relation between the inclusive and diffractive structure functions [12, 33],

$$
F_{2}^{D(3)}\left(\xi, \beta, Q^{2}\right) \sim \frac{1}{\ln Q^{2}} F_{2}\left(x=\xi, Q^{2}\right)
$$

which is in broad agreement with data [30]. For fixed momentum transfer $Q^{2}$ and diffractive mass $M$, both structure functions have the same dependence on the $\gamma^{*} p$ center-ofmass energy $W$. The factor $1 / \ln Q^{2}$ reflects the suppression of small colour dipoles in diffractive scattering.

The dependence of the diffractive structure functions on $\xi$ is not affected by the perturbative QCD evolution, contrary to the $x$ dependence of the inclusive structure functions, and therefore a genuine non-perturbative property of the proton. Hence, Eq. (39) can only hold as long as the effect of the perturbative evolution can be approximated by a single $\ln Q^{2}$ factor. The $\xi$ dependence of the diffractive structure function then plays the role of the non-perturbative input for the inclusive structure function at some low scale $Q_{0}^{2}$.

One expects that, due to unitarity, diffractive and inclusive structure functions satisfy a relation similar to the one between elastic and total proton-proton cross section [34],

$$
\begin{aligned}
\sigma_{e l} & =\int d^{2} b(1-S(b))^{2}, \\
\sigma_{t o t} & =2 \int d^{2} b(1-S(b)),
\end{aligned}
$$

where $S(b)$ is the S-matrix at a given impact parameter $b$. Recently, it has been shown that this relation also holds for the diffractive and inclusive cross sections of a $q \bar{q}$ pair off the proton, if the diffractive cross section is defined by the colour singlet projection [35]. In the semiclassical approach, this relation can be read off from Eqs. (18) and (19) or, more explicitly, from Eqs. (29) and (30). After integration over $l_{\perp}^{\prime}$ in Eqs. (31) and (32), which yields $y_{\perp}=y_{\perp}^{\prime}$, one obtains for the cross sections of a $q \bar{q}$ pair with size $y$,

$$
\begin{aligned}
\sigma_{q \bar{q}}^{D}(y) & \propto \frac{1}{N_{c}} \int_{x_{\perp}} \int_{A} \operatorname{tr} W_{x_{\perp}}\left(y_{\perp}\right) \operatorname{tr} W_{x_{\perp}}^{\dagger}\left(y_{\perp}\right) \\
& =\Omega N_{c}\left(1-e^{-a y^{2}}\right)^{2} \\
\sigma_{q \bar{q}}^{i n c l}(y) & \propto \int_{x_{\perp}} \int_{A} \operatorname{tr}\left(W_{x_{\perp}}\left(y_{\perp}\right) W_{x_{\perp}}^{\dagger}\left(y_{\perp}\right)\right) \\
& =2 \Omega N_{c}\left(1-e^{-a y^{2}}\right) .
\end{aligned}
$$

Since the dependence of the saturation parameter $a$ on the varying thickness of the target has been neglected, the integration over the impact parameter $x_{\perp}$ (corresponding to $b$ in(40),(41) ) could be carried out yielding the geometric size $\Omega$ as overall factor. It will be interesting to extend these considerations to more complicated partonic fluctuations. 
In the coming years experiments at HERA will provide detailed information about diffractive final states, including charm and high- $p_{\perp}$ jets. Anticipating further support of the semiclassical approach by data, we can hope to learn a lot about the colour structure of the proton from a comparison of inclusive and diffractive DIS.

The content of this progress report is largely based on recent work with Thomas Gehrmann and Arthur Hebecker whom I thank for an enjoyable collaboration.

\section{References}

[1] ZEUS collab., M. Derrick et al., Phys. Lett. B315 (1993) 481;

H1 collab., T. Ahmed et al., Nucl. Phys. B429 (1994) 477

[2] For recent reviews and references, see

A. Hebecker, Diffraction in deep inelastic scattering, HD-THEP-99-12, hepph/9905226:

M. Diehl, Diffractive Interactions: Theory Summary, DIS'99, Zeuthen, DESY 99080, hep-ph/9906518

[3] For a discussion and references, see

R.K. Ellis, W.J. Stirling, B.R. Webber, QCD and Collider Physics, Cambridge University Press, 1996

[4] A. Milsztajn, M. Virchaux, Phys. Lett. B274 (1992) 221;

A.D. Martin, R.G. Roberts, W.J. Stirling, R.S. Thorne, Phys. Lett. B443 (1998) 301

[5] V.N. Gribov, L.N. Lipatov, Sov. J. Nucl. Phys. 15 (1972) 438, 675;

G. Altarelli, G. Parisi, Nucl. Phys. B126 (1977) 298;

Yu.L. Dokshitzer, Sov. Phys. JETP 46 (1977) 641

[6] J.C. Collins, Phys. Rev. D57 (1998) 3051

[7] L. Trentadue, G. Veneziano, Phys. Lett. B323 (1994) 201

[8] A. Berera, D.E. Soper, Phys. Rev D50 (1994) 4328

[9] A. Berera, D.E. Soper, Phys. Rev D53 (1996) 6162

[10] G. Ingelman, P.E. Schlein, Phys. Lett. B152 (1985) 256

[11] A. Donnachie, P.V. Landshoff, Phys. Lett. B191 (1987) 309

[12] W. Buchmüller, A. Hebecker, Nucl. Phys. B476 (1996) 203

[13] J.D. Bjorken, AIP Conf. Proc. No.6, eds. M. Bander et al. (AIP, New York, 1972) p. 151 ;

J.D. Bjorken, J.B. Kogut, Phys. Rev. D8 (1973) 1341 
[14] W. Buchmüller, M.F. McDermott, A. Hebecker, Nucl. Phys. B487 (1997) 283; ibid. B500 (1997) 621 (E)

[15] J.D. Bjorken, J.B. Kogut, D. Soper, Phys. Rev. D3 (1971) 1382

[16] O. Nachtmann, Ann. Phys. 209 (1991) 436

[17] A. Hebecker, Nucl. Phys. B505 (1997) 349

[18] W. Buchmüller, T. Gehrmann, A. Hebecker, Nucl. Phys. B537 (1999) 477

[19] L. Frankfurt, G.A. Miller, M. Strikman, Phys. Lett. B304 (1993) 1

[20] L. McLerran, R. Venugopalan, Phys. Rev. D49 (1994) 2233

[21] A. Hebecker, H. Weigert, Phys. Lett. B432 (1998) 215

[22] W. Buchmüller and A. Hebecker, Phys. Lett. B355 (1995) 573

[23] A.H. Mueller, CU-TP-937, hep-ph/9904404

[24] E. Gotsman, E. Levin, U. Maor, Phys. Lett. B425 (1998) 369

[25] K. Golec-Biernat, M. Wüsthoff, Phys. Rev. D59 (1999) 014017; DTP/99/20, hep$\mathrm{ph} / 9903358$

[26] N.N. Nikolaev, B.G. Zakharov, Z. Phys. C49 (1991) 607

[27] J. Bartels, J. Ellis, H. Kowalski, M. Wüsthoff, Eur. Phys. J. C7 (1999) 443

[28] F. Hautmann, Z. Kunszt, D.E. Soper, Phys. Rev. Lett. 81 (1998) 3333; CERNTH/99-154, hep-ph/9906284

[29] H1 Collab., C. Adloff et al., Z. Phys. C76 (1997) 613

[30] ZEUS Collab., J. Breitweg et al., Eur. Phys. J. C6 (1999)43

[31] H1 Collab., S. Aid et al., Nucl. Phys. B470 (1996) 3;

ZEUS Collab., M. Derrick et al., Z. Phys. C72 (1996) 399

[32] M. Glück, E. Reya and M. Stratmann, Nucl. Phys. B422 (1994) 37

[33] W. Buchmüller, Phys. Lett. B353 (1995) 335

[34] A.H. Mueller, Eur. Phys. J. A1 (1998) 19

[35] Yu.V. Kovchegov, L. McLerran, NUC-MN-99/2-T, hep-ph/9903246 